\title{
SUSTAINABLE DEVELOPMENT AND PLANNING IN THE CASE OF UNCERTAINTY IN DIGITAL ELEVATION MODELS
}

\author{
G.A. ACHILLEOS \\ Technical University of Crete, Greece.
}

\begin{abstract}
The existence of elevation errors in Digital Elevation Models (DEMs) is usually ignored during spatial analysis of problems concerning planning through a sustainable sense of development. Examples of such analyses are: slope analysis for site selection, designing infrastructure works on physical terrains, 3D calculations on a basic surface like the terrain, sitting and zoning definition through elevation, etc. As a result, conclusions are extracted, decisions are made and actions are designed and executed, while the problem is examined theoretically, away from reality. This article describes the attempt to introduce a new model, the Digital Elevation Error Model, which incorporates elevation uncertainty and fuzziness, and accompanies a DEM, uniquely. The use of uncertain terrains combined with a probabilistic soft-decision theory, eliminates the risk of taking decisions that do not apply to the real problem under examination. The research on this subject has shown deviations from the results, from those of a hard-decision theory, up to 20-50\% for volume measurements, area measurements, definition of boundaries, visibility calculations, etc. The absence of an integrated geographic information system, able to manage data uncertainty, makes it necessary to use alternative approaches to the problem but not the appropriate and scientific ones.

Keywords: coastal zone, Digital Elevation Models, elevation errors, uncertain volume calculation, uncertainty, visibility analysis.
\end{abstract}

\section{INTRODUCTION}

Binary logic (or Boolean logic) is insufficient to represent and manage spatial data with their uncertainty and inaccuracy. This comprises a remarkable difficulty in cases of facing geometric problems. Furthermore, attempting to plan sustainable solutions through a conventional geographic information system (GIS), in combination with the insufficiency of the "YES or NO" decision practice allows doubts concerning the results. The theory of Probabilities and the theory of Fuzzy Logic comprise/constitute an important assistant and contributor toward this direction. Data are accompanied by their accuracy and are managed through Spatial Analysis applications and modules. The scope is to access result and decision having the knowledge of the inaccuracy and uncertainty that is included.

As it concerns cases and projects of sustainable planning and development, this research shows that the results need attention. This is due to the fact that planning for development, through an uncertain Digital Elevation Model (DEM), describing the real terrain, leads to false results. Based on these false results, scientists take decisions that have a high level of uncertainty and risk. This risk level is a function of the data they use and the decision procedure they follow.

The introduction of a new concept is attempted in this paper. This concept is the Digital Elevation Error Model (DEEM). This model in combination with the traditional DEM, introduces the Uncertain DEM (UDEM). Further on, the impact of this model in area and volume calculations, in boundary definition, and in cross section designs on terrain, is examined in order to understand the size of the impact on the results. 


\section{UNCERTAIN DIGITAL ELEVATION MODELS}

\subsection{The concept of a UDEM}

The meaning of a UDEM consists of a data structure of uncertain altimetry. This structure leads to the incorporation of elevation data uncertainty management, within a GIS.

Most of the GISs do not include abilities and modules for uncertain data management.

The uncertainty of an elevation structure is propagated into secondary products, resulting from the analysis of a DEM (slopes, areas, volumes, aspects, etc.).

Scientists dealing with the problem of uncertainty within the elevation of a DEM tried to deal with it in many ways. They tried to include these elevation errors within the analysis. Kraus et al. [1], Leyk et al. [2], Ravibabou et al. [3], Oksanen et al. [4], Aguilar et al. [5], Weng [6] and many more scientists have tracked down the problem of errors within DEMs and have proceeded to investigate these errors as well as their impact when elevations are used.

Professor Fisher in his extended work [7, 8] has used the RMS of the elevations within a DEM. Using a Monte Carlo approach, he simulated an error for every point of the terrain described by the DEM. This simulated error was then used to calculate secondary products, incorporating fuzziness.

In other extended works [9-13] the elevation errors were estimated using estimation models and the error propagation law [14]. The errors here were estimated for each pixel of the DEM and furthermore, they were propagated in secondary products, such as view sheds, volume calculations and area definitions.

Wechsler [15-17] has examined systematically the elevation error as well as its distribution within a DEM and investigated the impact of these errors in cases of using elevation data from such a DEM.

As can be seen, there is an attempt to define the elevation error within an elevation structure, regarding its size as well as its distribution. This attempt leads toward a data structure including elevation and its error for every defined point of space. This data structure can be then exploited in order to propagate the uncertainty existing within data. This data structure is defined in this paper as a UDEM and the error data structure as a DEEM.

The UDEM provides the researcher with the ability to choose the confidence level he wishes to work with, and this confidence level is usually compatible with the accuracy offered by the elevation data in use. Simultaneously, the researcher accepts the risk level he wishes to be introduced into his research and consequently into the final results, in case these results are applied in practice.

This transformation from a UDEM to a conventional one, allows the use of the existing GISs for analyzing the elevation data. The errors are embodied within data.

\subsection{The Digital Elevation Error Model}

The DEEM is a data structure model, incorporating the uncertainty contained within the elevation information. In combination with the DEM (Fig. 1), it is based on the acceptance of the normal distribution as the theoretical distribution describing the elevation error. The acceptance of the normal distribution has not been proved mathematically but it has been empirically confirmed and widely accepted [14]. The normal distribution, which is described by a mean value and a standard deviation value, covers $99.9 \%$ of the data within the range of values (Fig. 2):

$$
\begin{gathered}
\bar{x} \pm 3 \cdot \sigma_{x} \\
P\left(H<\bar{x}+3 \cdot \sigma_{x}\right)=\sim 100 \% \\
P\left(H>\bar{x}-3 \cdot \sigma_{x}\right)=\sim 100 \% \\
P\left(H<\bar{x}-3 \cdot \sigma_{x}\right)=\sim 0 \%
\end{gathered}
$$




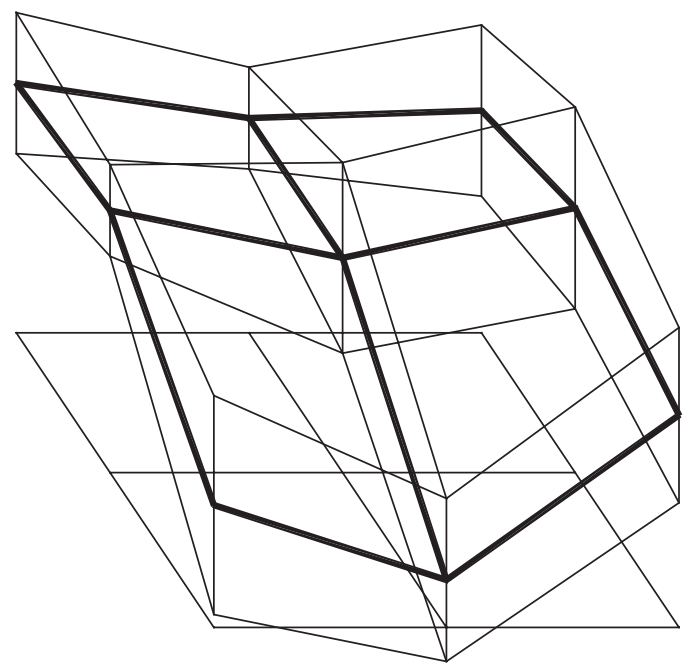

Figure 1: Perspective mode of an uncertain DEM and its DEEM.

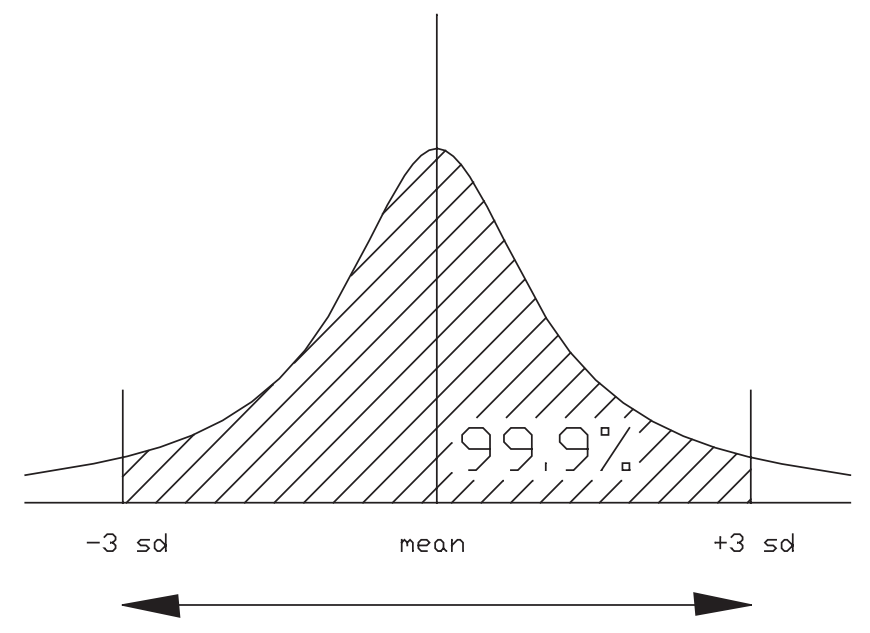

Figure 2: Probability function.

The probability function, giving the bell-shaped graph (Fig. 2), is (1):

$$
f(x)=\frac{1}{\sqrt{2 \cdot \pi}} \cdot \frac{1}{\sigma} \cdot e^{\left\{-\frac{(x-\bar{x})^{2}}{2 \cdot \sigma^{2}}\right\}}
$$

The cumulative probability function (or frequency function), giving the S-shaped graph (Fig. 3a and b), is (2):

$$
F(x)=\int_{\infty}^{x} f(x)=\int_{\infty}^{x} \frac{1}{\sqrt{2 \cdot \pi}} \cdot \frac{1}{\sigma} \cdot e^{\left\{-\frac{(x-\bar{x})^{2}}{2 \cdot \sigma^{2}}\right\}}
$$



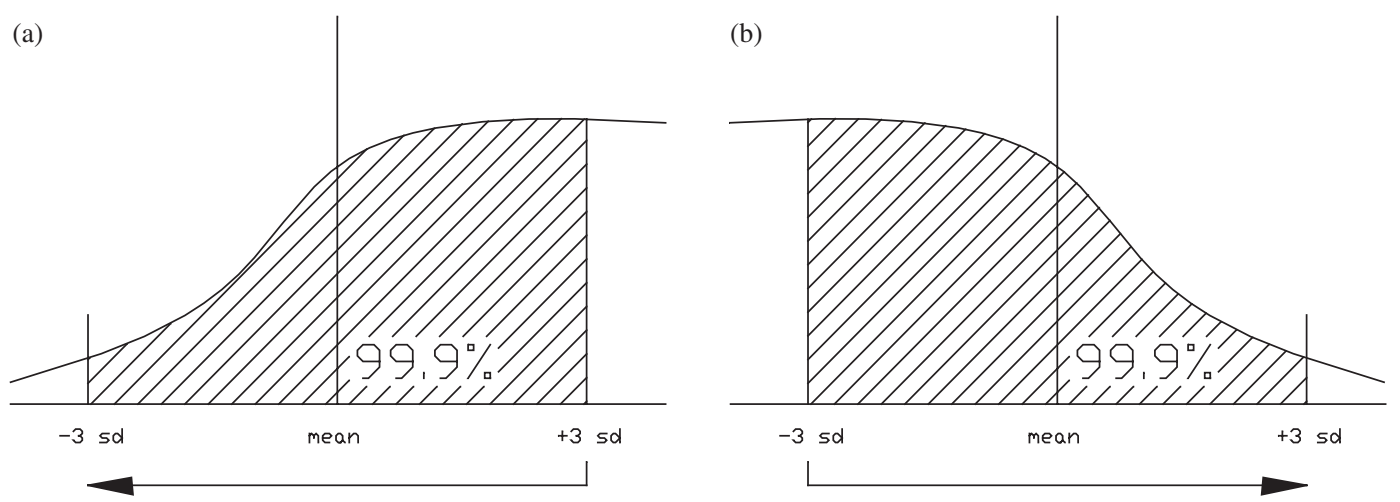

Figure 3: (a, b) Cumulative probability function.

The main issue here is the way this elevation error is determined and thus, the way the DEEM is constructed.

A recent extended work, which has been mentioned in the previous section, shows the procedure of producing a DEM using a topographic map [9, 10, 18, 19]. It also shows the way of producing the corresponding DEEM following the DEM production procedure and propagating the initial errors within this procedure.

Other approaches introduce the elevation error during the procedure of the elevation calculation recording in a structured database (i.e., automatic DEM generation in photogrammetry, satellite radar systems, etc.).

These elevation errors constitute the variation of the elevations in a DEM (or in other words, their standard deviation, the needed quantity for the DEEM definition). This variation is not a result that comes out of elevations and it is not a function of some kind of local processing of elevations, aiming to produce local uncertainty.

The DEM construction nowadays is no longer a time-consuming procedure. Thanks to informatics and computer technology, a DEM is constructed in a few seconds to a few minutes. The analysis of errors in order to achieve the uncertainty of this DEM, is more complicated (if this uncertainty is not a result of the DEM production - recording procedure). Nevertheless, the time required for the acquisition of DEM uncertainty in combination with the existing technology setup make it possible for everyone to achieve the uncertainty of the DEM.

\subsection{From the probabilistic to a deterministic DEM}

The transition from an uncertain, probabilistic DEM to a deterministic one takes shape based on the confidence level the researcher selects (or in other words, based on the risk level the researcher is prepared to undertake). The critical value of the elevation, according to the confidence level, is calculated from the cumulative probability function, as the value for which the Riemann integral, with that elevation value as a limit, gives an area equal to the confidence level. The critical value is then used within the procedure. The determination of this critical value, takes place for every pixel of the DEM, resulting in a new DEM that represents the selected confidence level. A selection of a different confidence level provides a different DEM.

Figures 4 and 5 present in order, the DEM, its DEEM and the DEMs for the confidence levels $75 \%, 90 \%$, and $99 \%$. 

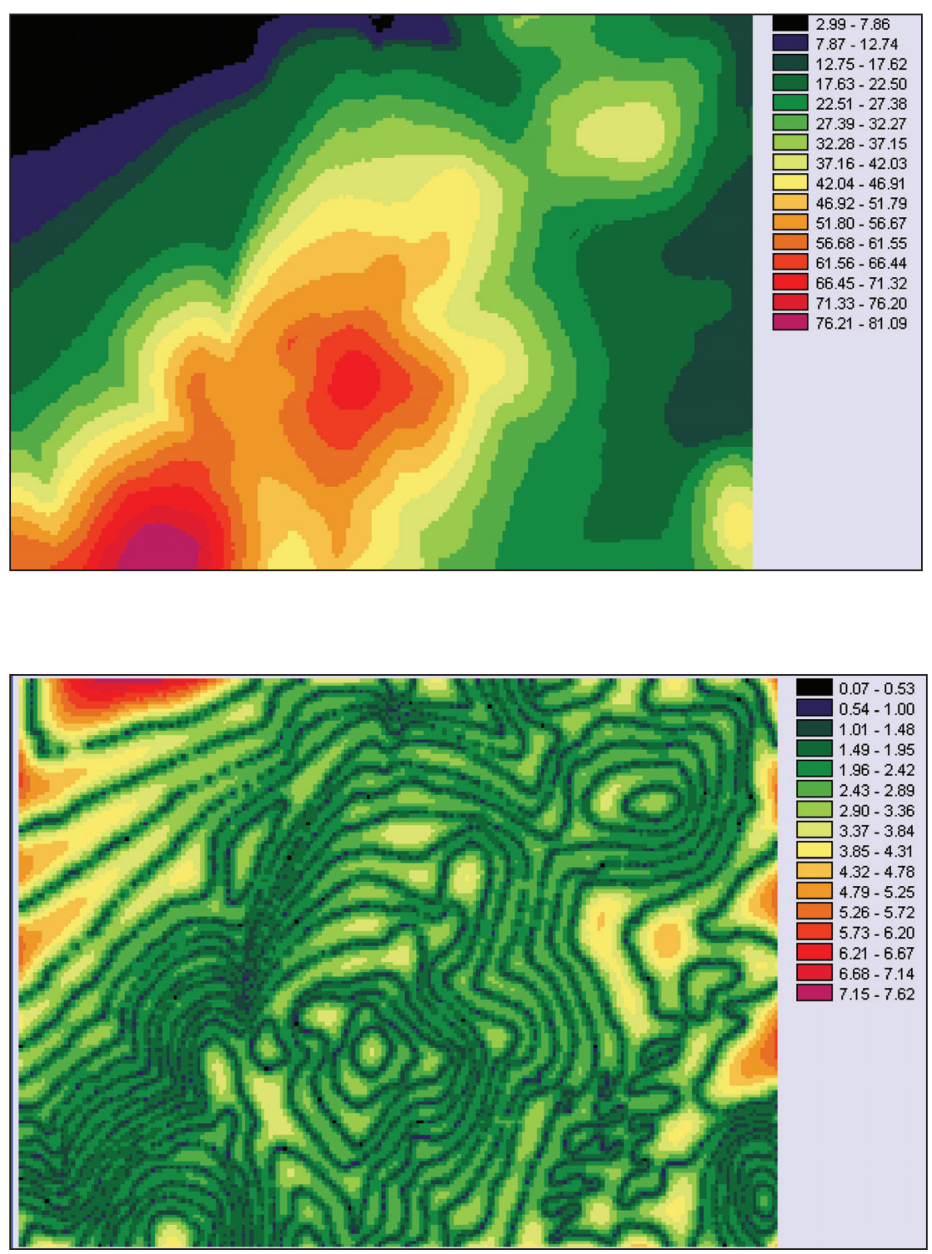

Figure 4: DEM and its DEEM.

\section{SUSTAINABLE PLANNING AND DEVELOPMENT THROUGH UNCERTAINTY}

3.1 The importance of a sustainable planning and development

The term "sustainable" introduces the meaning of duration and long living through time. Planning interferences in space with the motive of improvement and development, unavoidably someone is charged with the responsibility of taking into account the sustainability of his plans and actions.

Actions that are taken and affect the environment should not influence and complicate human lives. Especially, these actions should not put human lives in danger. Otherwise, these actions do not correspond to the meaning of sustainability. Risky planned projects and actions containing low level of safety and hygiene do not incorporate requirements for a sustainable development.

The meaning of uncertainty influences this trend for a sustainable thought. It covers a variety of factors, such as data, models, conditions, relationships between conditions, etc. 
(a)

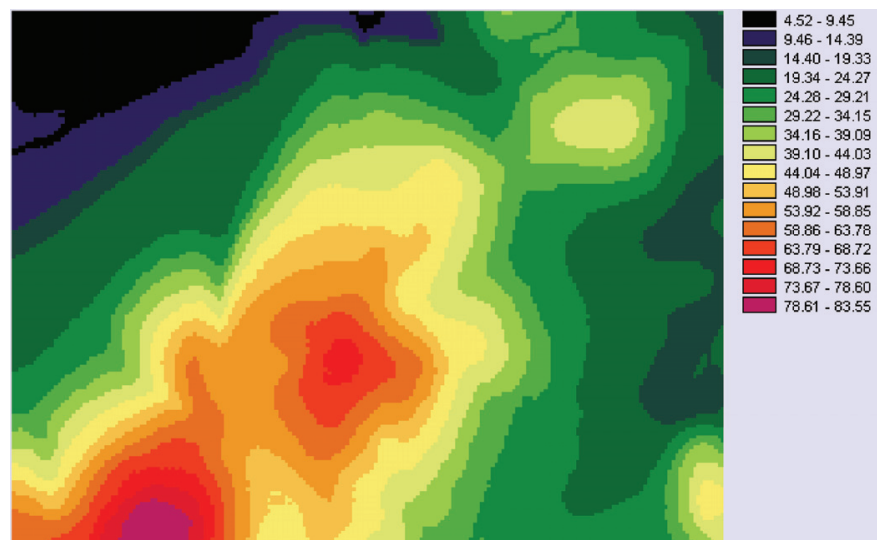

(b)

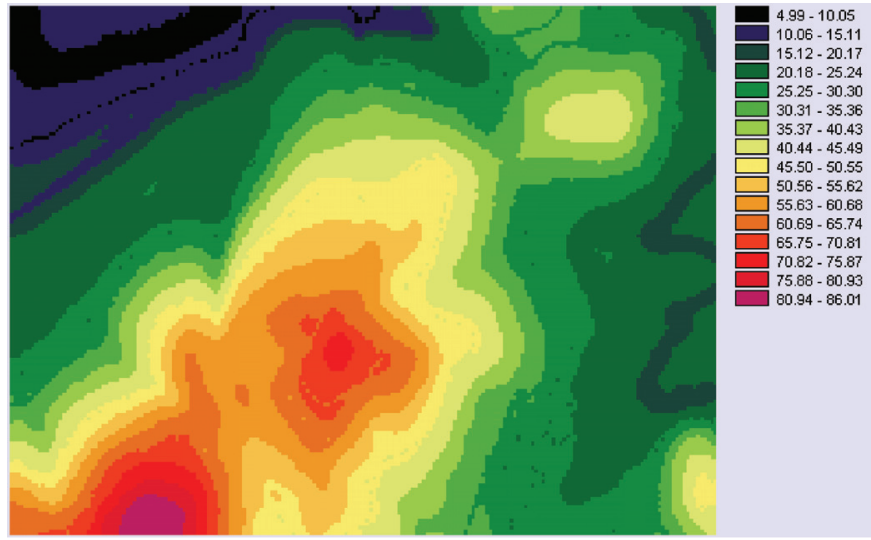

(c)

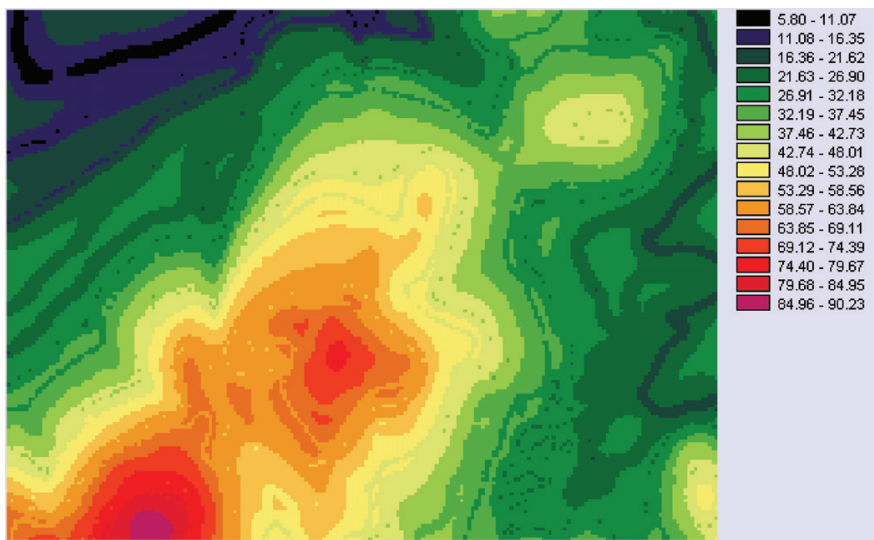

Figure 5: DEM for certain confidence levels: (a) 75\%; (b) 90\%; and (c) 99\%. 
The basic component of planning is the set of data that is used. This set of data usually contains stochastic errors and the only available element is an estimation of their size and distribution. Due to this fact, the incorporation of uncertainty within the procedure of planning is unavoidable.

Therefore, the target is not the avoidance of errors and the introduced uncertainty, but the incorporation of them within the procedure.

\subsection{Influence of using uncertain terrains in analysis}

The use of uncertain DEMs as representations of terrain influences the analysis within a procedure of sustainable planning and development. As a result, there are contestations concerning the outcomes of this analysis, especially by specialists believing on a soft-decision procedure and not a hard one.

The conventional analysis, using binary logic, reaches a decision that differs importantly from a decision resulting from an alternative analysis, which is based on theories such as the uncertainty theory, the fuzzy logic theory and the theory of probabilities.

The determination of geometrical characteristics, which may or may not have a geometrical hypostasis but are determined through a digital system using a DEM, requires the consideration of any elevation error included in this DEM. The influence is immediately observed in area and volumetric calculations, site selections, zone and boundary definitions, 3D calculations, etc.

The research presented in this article covers three case studies of analysis for sustainable planning: case study 1 concerning coastal zone boundary definition, case study 2 concerning capacity volume calculations for water tanks behind dams and case study 3 concerning the visibility analysis.

\section{CASE STUDIES}

\subsection{Case study 1 - coastal zone boundary definition}

The first case that can be managed through a DEM is the case of the coastal zone.

A well-accepted definition says that: "the coastal zone can be determined from a DEM as the elevation zone between the values of minus $10 \mathrm{~m}$ to plus $10 \mathrm{~m}$ from the sea level [20]." Based on this definition, the coastal zone of the study area is determined and marked as shown in Figs. 6 and 7. Figure 6 presents the binary calculated coastal zone while Fig. 7 presents the uncertain version.

Calculating the coastal zone in classical binary and uncertain format analyses (Figs. 8 and 9), it is observed that the probabilistic approach to the coastal zone presents larger sizes than the certain binary one. The black boundary lines in these figures (Figs. 8 and 9) show the extension of the uncertain coastal zone. The difference for the certain area is $30.14 \%$. This difference is serious and noticeable in cases where there is a planning policy for managing and developing this zone [11].

\subsection{Case study 2 - capacity volume calculations}

The certain case study covers a fictitious preliminary study of an artificial water tank, resulting from the construction of a dam. The aim of the preliminary study is the estimation of the total capacity of the 3D-shaped solid the water will fill. Further on, the selection of the certain place and solution specifications are compared with other alternative places and different specifications for the project, in order to achieve the final selection. 


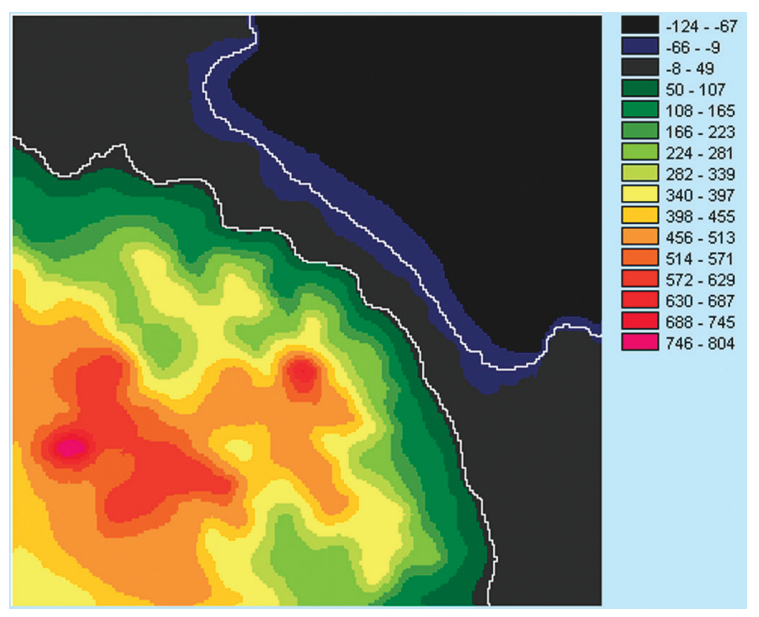

Figure 6: Topography.

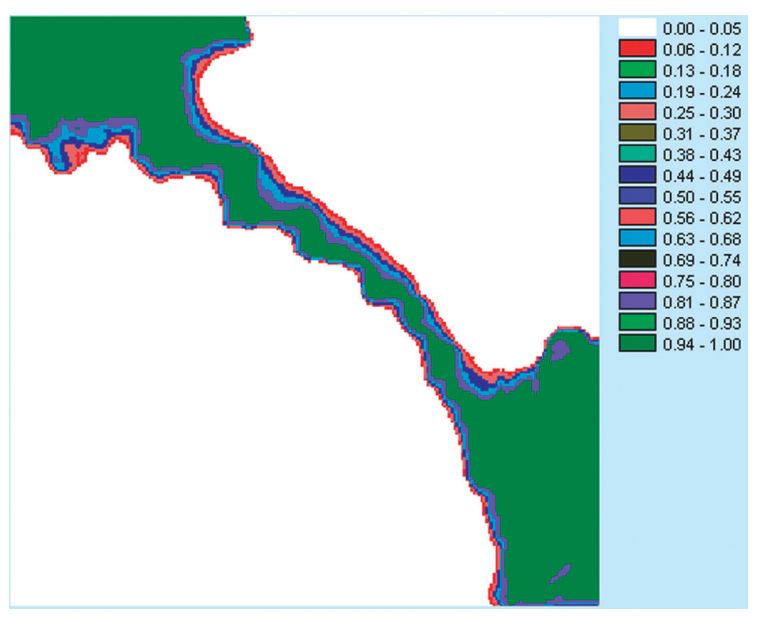

Figure 7: Uncertain coastal zone $(-10 \mathrm{~m}<\bullet<+10 \mathrm{~m})$.

The elevation data used for the selection of this position of the dam, and the volumetric calculations for the capacity of the tank, come from topographic 1:20,000 scale maps. The contour interval is $5 \mathrm{~m}$ for the cases of a preliminary study and an Environmental Impact Assessment study. These data of course are not appropriate for a final study. The elevation accuracy provided is $\pm 2.5 \mathrm{~m}$. The elevation level of the dam top and its overflow position is $340 \mathrm{~m}$.

The DEM with the dam's selected axis are presented in Fig. 10. Binary analysis provides the area that stands higher than $340 \mathrm{~m}$ (Fig. 11).

Using the membership function, one can estimate the area which presents a probability of $75 \%$ that is altimetricaly higher than $340 \mathrm{~m}$ (the membership function presents values higher than 0.75). The same area can be estimated for a probability of $90 \%$ or $99 \%$. These areas can be seen in 


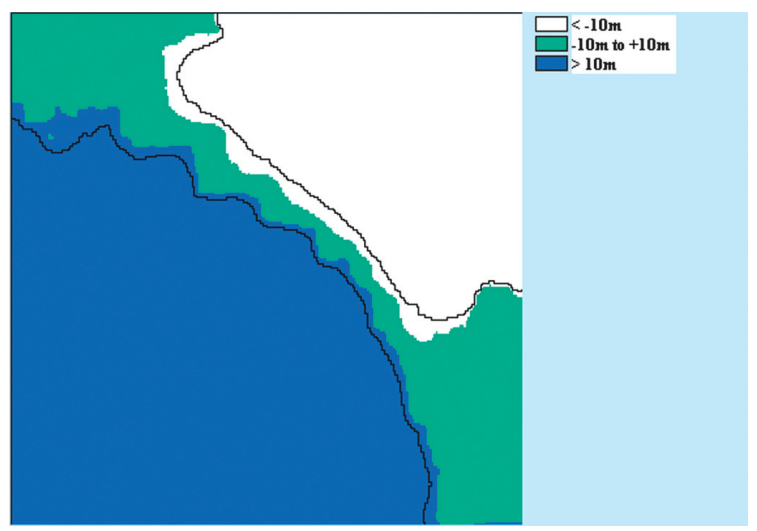

Figure 8: Binary coastal zone.

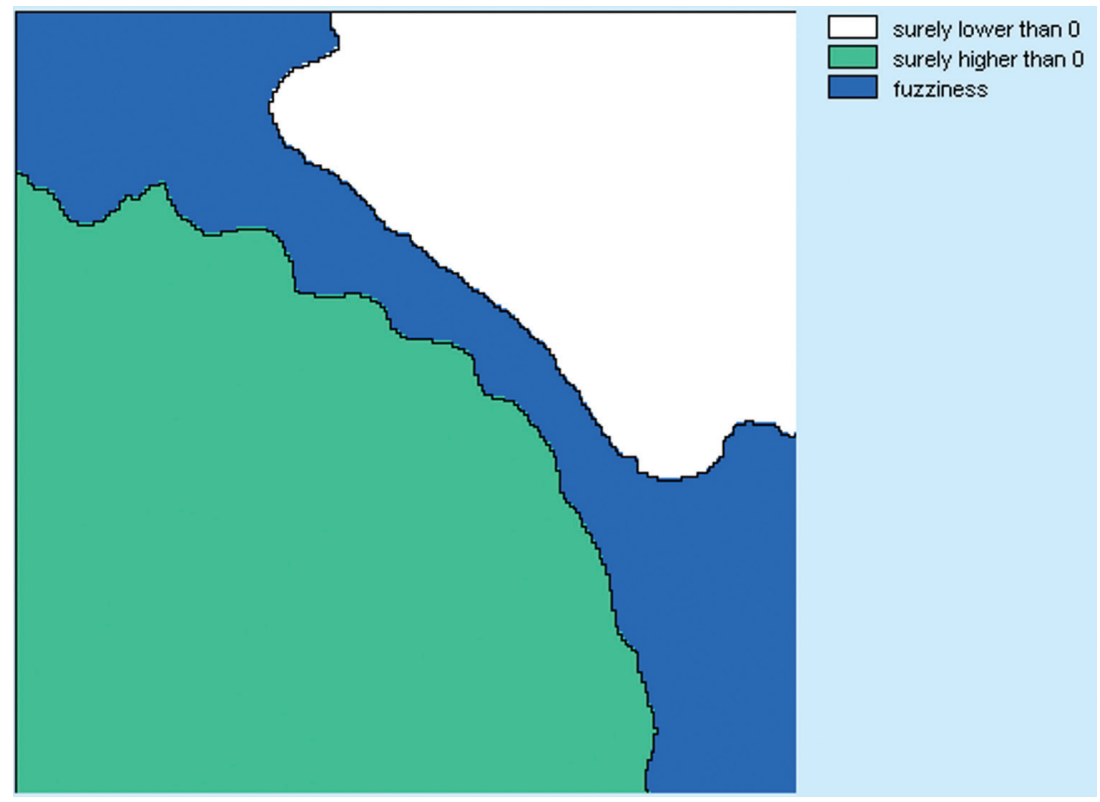

Figure 9: Uncertain coastal zone.

Figs. 12-15. It can be realized from these figures that the greater the confidence level, the greater the proportion of the study area which is altimetricaly higher than $340 \mathrm{~m}$. This increment in the area of interest introduces points for water overflow (water escape points), which arithmetically increase with the increment in the confidence level.

The volumetric calculations are based on the assumption of the geometric volume estimation of solid shapes. This is the product of the base of the shape multiplied by its height. In the certain case, the height of the solid shape is considered to be the mean elevation difference, presented in the area of the tank (base). 


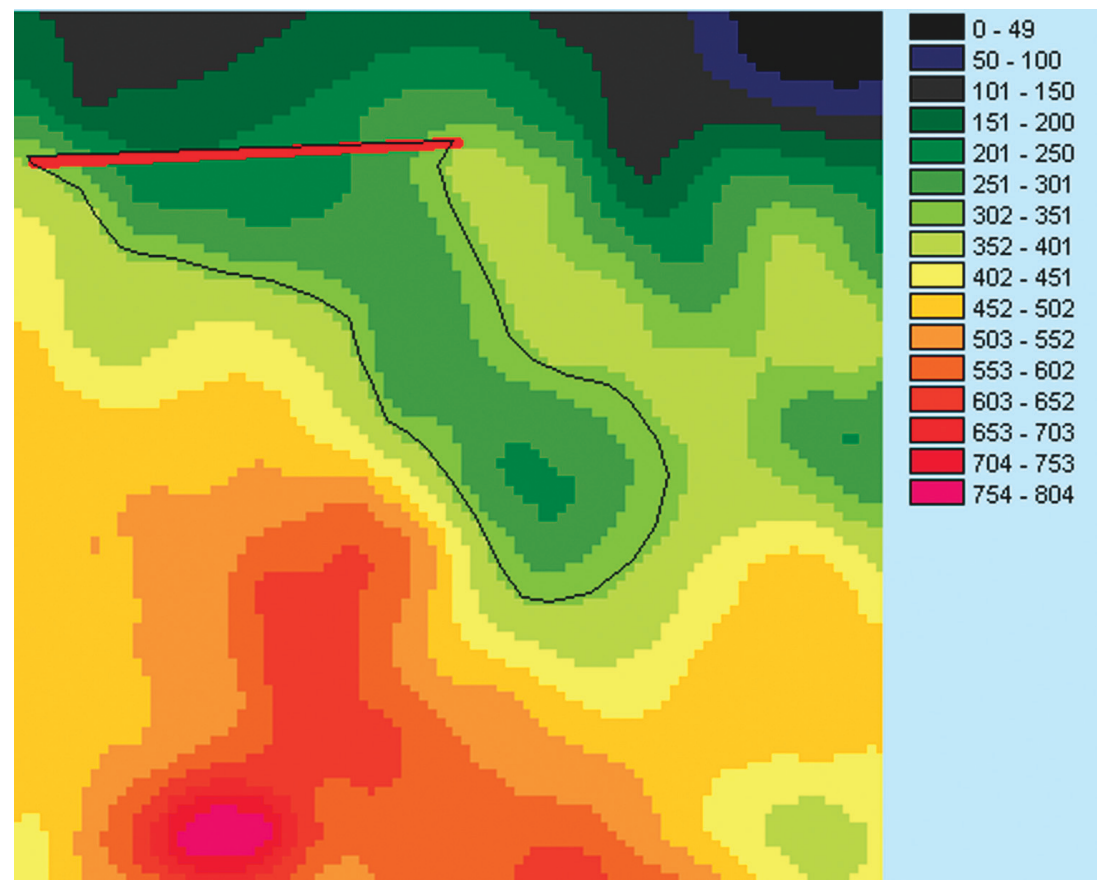

Figure 10: DEM.

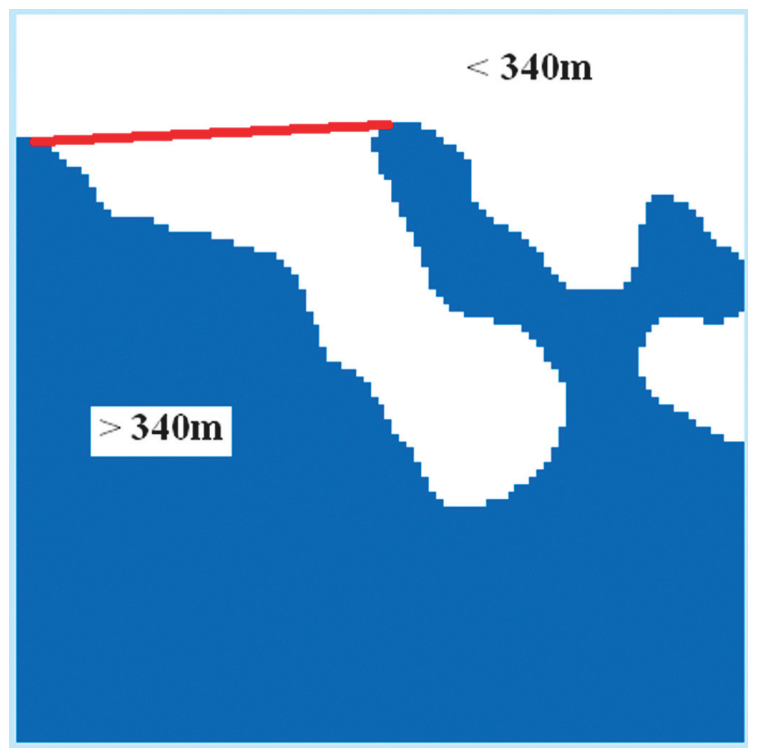

Figure 11: Area elevations $>340 \mathrm{~m}$ (binary result). 


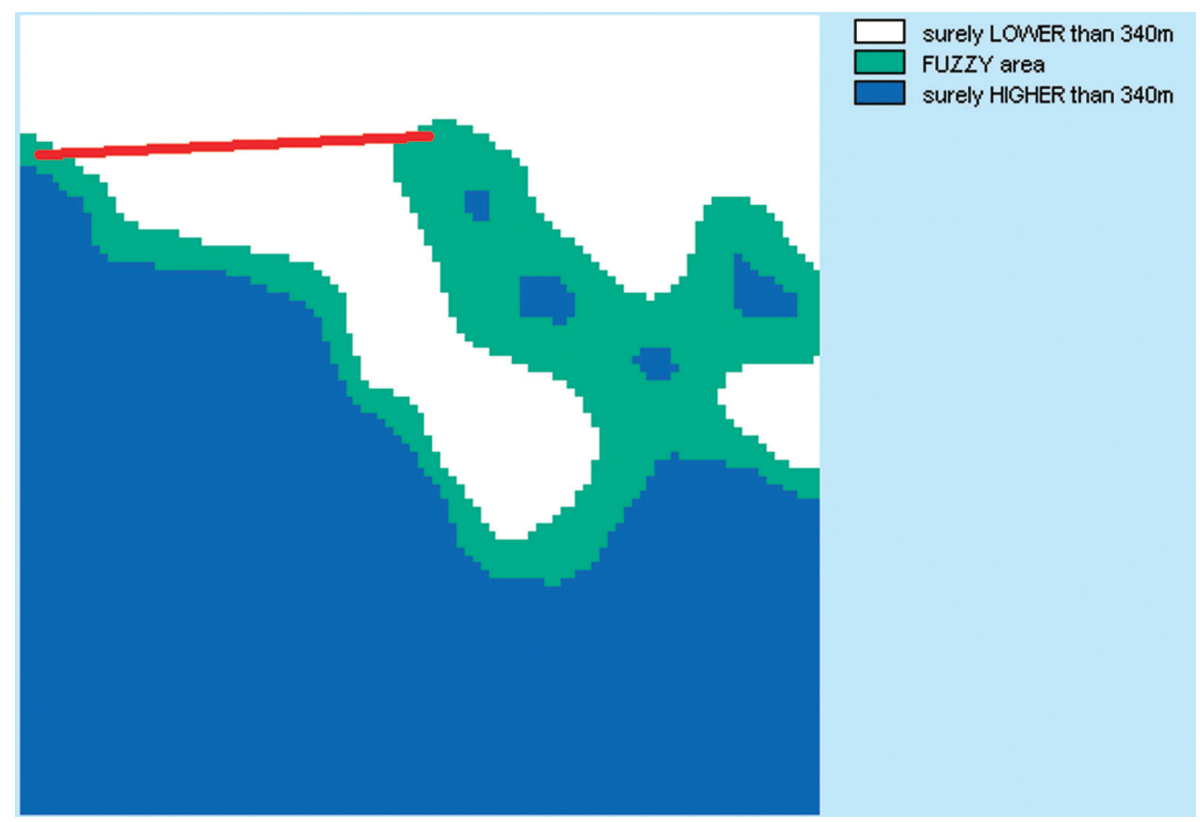

Figure 12: Uncertain area (midtone: surely <340 m, dark: surely >340m, white: uncertainty).

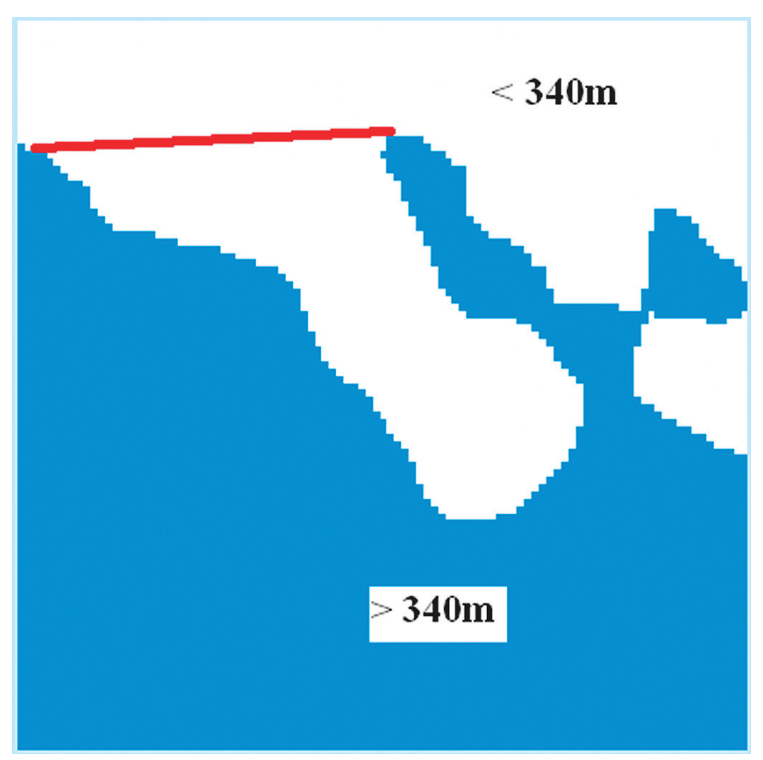

Figure 13: Area altimetrically higher than $340 \mathrm{~m}(P>75 \%)$. 


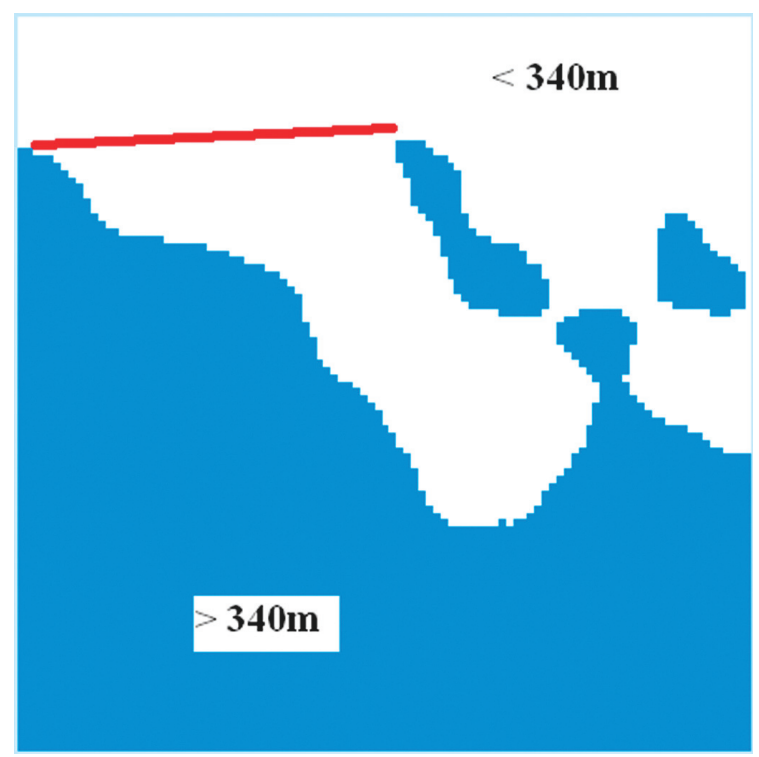

Figure 14: Area altimetrically higher than $340 \mathrm{~m}(P>90 \%)$.

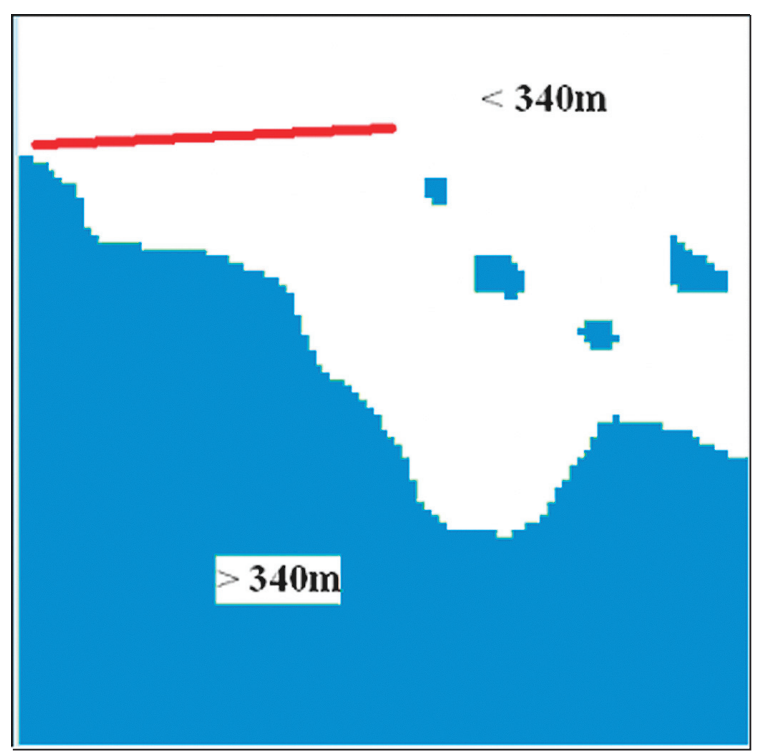

Figure 15: Area altimetrically higher than $340 \mathrm{~m}(P>99 \%)$. 
This assumption is applied due to the problem presented in this research, of the underestimation of the volume, using the analytical tools of the GIS packages. This problem is a result of the fact that some points of the area of interest, with elevations lower than the dam top $(340 \mathrm{~m})$, are included in the area of base for certain values of the confidence level. These points give negative elevation differences, which seriously influence the final calculation of the capacity of the water tank.

The approach this application follows is examined concerning its correctness.

The results of the area and the capacity of the water tank (volume) under consideration are presented in Table 1 . The research covers the confidence levels of 75\%, 90\% and 99\%. The observed deviations from the water tank capacity are within the range of $10-40 \%$.

\subsection{Case study 3 - visibility analysis}

The visibility calculation is one of the basic procedures existing in GIS, which have the ability to manage elevation data.

Therefore, as the process of vision is closely/strongly linked to the "everyday" needs of the society, attention should be paid in order to ensure an accurate calculation of visibility.

A designed algorithm, GAVOS, uses the elevations from a DEM and the variations of these elevations from a DEEM, and calculates an estimation of the probability that a Target Point (TP) is visible from a View Point (VP) (Table 2). We select the best-fitted Line of Sight (LoS) (Fig. 16), which gives the maximum probability that visibility exists between TP and VP.

The probability of line $A B$ existing ( $B$ is visible from $A$ ) is the product of probabilities, considering that these events are independent:

$$
\begin{gathered}
P A, P i_{1}, P i_{2}, \ldots, P i_{15}, P B \\
P=P(A) \cdot P\left(i_{1}\right) \cdot P\left(i_{2}\right) \cdots P\left(i_{15}\right) \cdot P(B)
\end{gathered}
$$

Table 1: Research results of area and volume.

\begin{tabular}{lccccc}
\hline $\begin{array}{l}\text { Confidence } \\
\text { level } \\
(\%)\end{array}$ & $\begin{array}{c}\text { Area } \\
\left(1000 * \mathrm{~m}^{2}\right)\end{array}$ & $\begin{array}{c}\text { Mean } \\
\text { elevation } \\
(\mathrm{m})\end{array}$ & $\begin{array}{c}\text { Elevation } \\
\text { difference } \\
(\mathrm{m})\end{array}$ & $\begin{array}{c}\text { Solid shape } \\
\text { volume } \\
\left(1000 * \mathrm{~m}^{3}\right)\end{array}$ & $\begin{array}{c}\text { Deviation } \\
(\%)\end{array}$ \\
\hline BIN & 3547.5 & 280.83 & 59.17 & 209905.5 & - \\
75 & 3897.5 & 286.97 & 53.03 & 230615.0 & 9.9 \\
90 & 4267.5 & 292.81 & 47.19 & 252507.9 & 20.3 \\
99 & 5055.0 & 303.98 & 36.02 & 299104.3 & 42.5 \\
\hline
\end{tabular}

Table 2: Variation of probabilities.

\begin{tabular}{lc}
\hline Elevation error & Maximum probability to see \\
\hline 2.00 & 96.40 \\
3.00 & 78.21 \\
4.00 & 53.12 \\
5.00 & 40.71 \\
6.00 & 27.16 \\
\hline
\end{tabular}




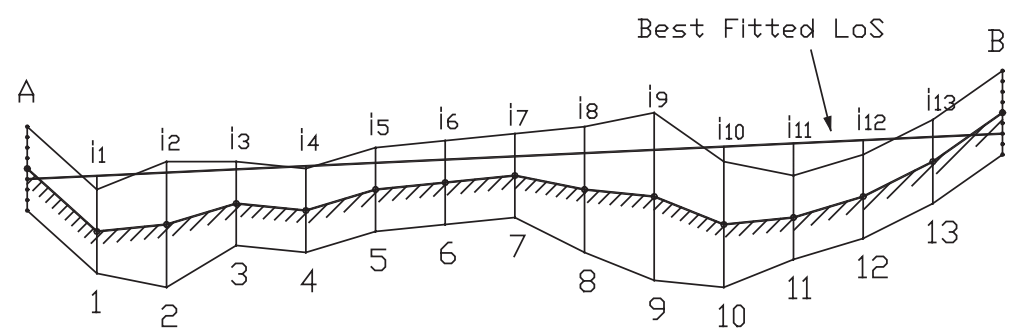

Figure 16: Best fitted LoS.

\section{REMARKS AND COMMENTS}

As can be realized from this work, spatial data uncertainty should be incorporated within the calculation procedures. Spatial data analysis systems (such as GISs) should be extended to cope with uncertainty of data and manage this uncertainty using their modules.

The analysis of elevation uncertainty has shown that the errors in the results are important and the risk level of a researcher who bases his work on these results is quite high. It seems that much work can be done, having no substance in reality if uncertainty is bypassed.

Coastal area calculation presents a $30 \%$ error with a confidence level of $95 \%$. This $30 \%$ deviation can mislead the planners of a coastal zone development project to decide and act wrongly. The consequences could be serious

The same danger exists within the water tank volume calculation, as a $42 \%$ deviation from a conventional approach (binary decision) could be catastrophic for such projects. An inconvenient project position could be decided, putting people and properties in danger in case of a dam stability failure.

In the case of visibility analysis, it might look as if there are no serious consequences of a wrong estimation. Nevertheless, impacts here could be as well important. If someone is locating fire prevention towers or army observation points, then an $80 \%$ "possibility to see" or a "30\% possibility to see" in relation to the " $100 \%$ possibility to see" through a binary calculation approach seems like a total failure.

The remaining main problem for approaching decisions with a percentage of confidence is the estimation of the UDEM (or the DEEM, in other words). As already mentioned, an accurate estimation of the DEEM, in relation to the proper data analysis tools, contributes toward this direction. An approach to a DEEM generation through objective procedures is presented in a $\mathrm{PhD}$ dissertation [10]. An elevation error is calculated statistically for every elevation in a DEM.

Planning in a sustainable manner and targeting toward development assumes that the basis of the work is certain and undoubted. The spatial database on which one is setting up all ideas should not allow the incorporation of high risk levels.

\section{REFERENCES}

[1] Kraus, Y., Shoshani, U. \& Doytsher, Y., Precise coordinate LIS for improving planning and land registration processes in modern real estate markets. FIG Working Week 2007, Strategic Integration of Surveying Services, Hong Kong SAR, China, 13-17 May 2007.

[2] Leyk, St., Weibel, R. \& Boesch, R., A conceptual framework for uncertainty investigation in map-based land cover change modelling. Transactions in GIS, 9(3), pp. 291-322, 2005.

[3] Ravibabou, M. \& Jain, K., A web-based survey on digital elevation models. Journal of Geographic Information Sciences, 12(1), pp. 34-37, 2006. 
[4] Oksanen, J. \& Sarjakoski, T., Error propagation of DEM-based surface derivatives. Computers \& Geosciences, 31(8), pp. 1015-1027, 2005.

[5] Aguilar, F.J., Aguilar, M.A. \& Aguera, F., Accuracy assessment of digital elevation models using a non-parametric approach. International Journal of Geographical Information Science, 21(6), pp. 667-686, 2007.

[6] Weng, Q., Quantifying uncertainty of digital elevation models derived from topographic maps. Advances in Spatial Data Handling, eds D. Richardson \& P. van Osterom, Springer-Verlag: New York, pp. 403-418, 2002.

[7] Fisher, P.F., Algorithm and implementation uncertainty in viewshed analysis. International Journal of Geographical Information Systems, 7(4), pp. 331-347, 1993.

[8] Fisher, P.F., An exploration of probable viewsheds in landscape planning. Environment and Planning B: Planning and Design, 22, pp. 527-546, 1995.

[9] Achilleos, G., Digital elevation models: elevation error. The error due to the application of the affine transformation on the contour lines. Technika Chronika - TEE, Section A, 17(3), pp. 27-46, 1997 (in Greek).

[10] Achilleos, G., Geographic Information Systems: Investigating Errors in Processes of Digital Recording of Contours and Producing DEM, PhD Dissertation, Department of Rural and Surveying Engineering, NTUA, 2002 (in Greek).

[11] Achilleos, G., Definition of the coastal zone through a fuzzy digital elevation model. Proceedings of the Second Hellenic Conference: Management and Improvement of Coastal Zones. Athens, November 25-28, 2002 (in Greek).

[12] Achilleos, G., Volume calculation using fuzzy logic in a waste sanitary landfill design. 12th ArcGIS Users Conference, Athens, 6-8 November 2002 (in Greek).

[13] Achilleos, G., Considering elevation uncertainty for managing probable disasters. Proceedings of the First International Symposium on Geo-information for Disaster Management, Delft, the Netherlands, March 21-23, pp. 251-263, 2005.

[14] Veis, G. \& Agantza-Mbalodimou, A., Error Theory and Least Square Method, Semester Notes, School of Rural \& Surveying Engineering, NTUA, Athens, 1989 (in Greek).

[15] Wechsler, S. \& Kroll, Ch., Quantifying DEM uncertainty and its effect on topographic parameters. Photogrammetric Engineering \& Remote Sensing, 72(9), pp. 1081-1090, 2006.

[16] Wechsler, S., Perceptions of digital elevation model uncertainty by DEM users. URISA Journal, 15(2), pp. 57-64, 2003.

[17] Wechsler, S., Uncertainties associated with digital elevation models for hydrologic applications: a review. Hydrology and Earth System Sciences, 11, pp. 1481-1500, 2007.

[18] Achilleos, G., Propagation of uncertainty within the affine transformation application on contours. Journal of Geographic Information Sciences, 12(2), pp. 79-91, 2006.

[19] Achilleos, G., Errors within the inverse distance weighted (IDW) interpolation procedure. Geocarto, i-First, pp. 1-21, 10 April 2008.

[20] St. Martin, K. (ed.), Applications in Coastal Zone Research and Management. Explorations in Geographic Information Systems Technology, Vol. 3, Clark Labs, UNITAR European Office, Switzerland, 1993. 